\title{
Screening par chromatographie liquide couplée à la spectrométrie de masse (LC-MS)
}

\section{Screening by liquid chromatography coupled with mass spectromety (LC-MS)}

Hans H. MAURER

Department of Experimental and Clinical Toxicology, University of Saarland D-66421 Homburg (Saar) - Germany

*Auteur à qui adresser la correspondance : Prof. Dr Hans H. MAURER, Department of Experimental and Clinical Toxicology, Institute of Experimental and Clinical Pharmacology and Toxicology, University of Saarland - D-66421 Homburg (Saar) - Germany Tel : +496841 1626050 - Fax : +496841 1626051 - E-mail : hans.maurer@uniklinik-saarland.de

(Reçu le 30 janvier 2005; accepté après modifications le 15 avril 2005)

\section{RÉSUMÉ}

Cet article passe en revue les procédures de screenings chromatographiques utilisant la chromatographie liquide couplée à la spectrométrie de masse pour la détection simultanée (urine ou sang) de plusieurs classes de médicaments ou de drogues importantes en toxicologie clinique, médicolégale ou en analyse anti-dopage, à l'aide d'une détection quadripolaire simple ou en tandem (LC-MS, LC-MS/MS). Ces deux techniques sont devenues des compléments idéaux à la chromatographie gazeuse couplée à la spectrométrie de masse (GC-MS), notamment pour la détection des substances plus polaires, thermolabiles et/ou actives à faible dose, et ce, tout particulièrement dans le plasma. La fragmentation n'est cependant pas très reproductible et l'ionisation peut être fortement réduite par le phénomène de suppression dû, entre autres, à la matrice co-éluante. Le coût de l'appareillage reste très élevé.

\section{MOTS-CLÉS}

Screening, quantification, médicaments, drogues, chromatographie liquide, spectrométrie de masse.

\section{SUMMARY}

This paper reviews chromatographic screening procedures for simultaneous detection of several drug classes relevant to clinical and forensic toxicology or doping control in urine or blood using liquid chromatography coupled with a single stage or tandem mass spectrometer (LC-MS, LC-MS/MS). Both techniques have shown to be ideal supplements to gas chromatography-mass spectrometry (GC-MS), particularly for detection of more polar; thermolabile and/or low-dosed drugs, especially in blood plasma. However; the fragmentation is not reproducible, the ionization may markedly be reduced by ion suppression caused e.g. by co-eluting matrix, and the costs of the apparatus are still very high.

\section{KEY-WORDS}

Screening, quantification, drugs, liquid chromatography, mass spectrometry. 


\section{Introduction}

Une analyse toxicologique pertinente est la base d'un jugement, d'une consultation et d'une expertise toxicologique de haute qualité. En toxicologie clinique, en toxicologie médico-légale et en contrôle anti-dopage, les composés qui doivent être analysés sont, la plupart du temps, inconnus. Par conséquent, avant toute quantification, la première étape est le dépistage (screening) et l'identification des composés sur lesquels l'intérêt va se focaliser (1). Deux types de stratégies de screening doivent être différenciées : d'une part le «screening ciblé» d'un nombre limité de composés connus (groupes des drogues d'abus ou de substances dopantes) et, d'autre part, le «screening général» d'un nombre illimité de substances comprenant les médicaments communs et les poisons, les substances rares et/ou inattendues voire même des drogues jusqu'ici inconnues (par exemple de nouvelles drogues de synthèse). Pour de tels screenings, des techniques de chromatographie sur couche mince (2) et de chromatographie en phase gazeuse avec les détecteurs conventionnels (3-6), ainsi que des techniques électrocinétiques $(7,8)$ ont été décrites. Elles ne sont cependant pratiquement plus employées. Actuellement, la chromatographie en phase gazeuse couplée à la spectrométrie de masse (GC-MS) et la chromatographie liquide à haute pression (HPLC) couplée à un détecteur à barrette de diodes (DAD) sont utilisées (1). La GC-MS a un excellent pouvoir de séparation et fournit des spectres de masse riches en information, très reproductibles, permettant l'utilisation de différentes bibliothèques de référence totalisant plus de 500000 spectres. Les appareillages peu coûteux, de type benchtop, sont entièrement adaptés au screening global effectué sur l'urine. L'utilisation de l'HPLC couplée à un spectromètre de masse à simple quadripôle ou en tandem (LC-MS, LCMS/MS) est encore assez limitée, mais quelques applications semblent prometteuses $(1,9-26)$.

Malheureusement, la LC-MS et la LC-MS/MS possèdent un pouvoir de séparation assez limité et il existe un risque important de suppression d'ions. En raison d'un contenu en information et d'une reproductibilité assez variables, seules quelques bibliothèques spectrales «faites maison» sont disponibles en LC-MS. D'autre part, cet appareillage reste très coûteux.

Néanmoins, la LC-MS et la LC-MS/MS sont des techniques idéales pour le screening ciblé et le dosage validé de médicaments et/ou de drogues (plutôt polaires) dans le plasma.

Dans les paragraphes qui suivent, différentes procédures de screening pour la détection simultanée de plusieurs classes de médicaments et/ou de drogues sont passées en revue, afin d'en préciser la position actuelle et d'en évaluer la place future en toxicologie clinique et médico-légale ou analyse anti-dopage. Les articles publiés avant 2000 ont déjà fait l'objet d'une revue (27) et ne seront pas pris en compte ici. Pour des raisons de place, seules ont été considérées les procédures portant sur l'analyse simultanée de plusieurs classes de médicaments et/ou de drogues dans líurine ou le sang.

\section{Choix de l'échantillon bio- logique}

Le sang (plasma ou sérum) est l'échantillon de choix pour toute détermination quantitative. Malgré tout, un screening sur un dérivé sanguin peut être envisagé et réalisé, pour autant que les concentrations des analytes soient suffisamment élevées. C'est particulièrement utile si l'on a uniquement accès à des échantillons sanguins et/ou si les procédures permettent le screening et la quantification simultanés $(10,15,16,18,21,24-26$, 28). Cependant, dans la mesure où les concentrations des médicaments sont relativement élevées dans les urines et, où les échantillons biologiques peuvent être prélevés de manière non traumatique, l'urine reste toujours un milieu de choix pour le screening et l'identification des médicaments ou des poisons inconnus par GC-MS (1) mais également par LC-MS (1, 11, 12, 23, 29-31). Pour l'analyse anti-dopage, tant chez l'homme que chez le cheval, l'urine est l'échantillon de screening (23). Dans le cas de la LC-MS ou de la LCMS/MS, la possibilité d'une suppression d'ions due à la co-élution de la matrice urinaire doit être investiguée sinon exclue. Pour terminer, rappelons que l'analyse de l'urine peut être inadéquate lors d'investigations de cas médico-légaux ou de cas de surdosages aigus pouvant mener à une mort rapide.

\section{Préparation de l'échan- tillon biologique}

On a longtemps dit que la LC-MS était moins exigeante en termes de préparation de l'échantillon biologique. Cependant, une extraction plus ou moins sélective est importante pour l'analyse en LC-MS (32), tout particulièrement pour éviter les effets de suppression d'ions (33-38). Il est étonnant de lire que certaines procédures de screening urinaire par LC-MS ou LC-MS/MS avaient recours à une longue hydrolyse enzymatique des conjugués $(20,23,31,39)$. Une seule procédure de screening est basée sur l'injection directe de l'urine suivie d'une confirmation après préparation par extraction en phase solide (SPE) (40). La préparation de l'échantillon peut être effectuée par extraction liquide- 
liquide (LLE) à un pH auquel l'analyte est non ionisé ou par extraction en phase solide (SPE) précédée ou suivie d'étapes de purification. Le pré-traitement de l'échantillon pour la SPE dépend du type de liquide biologique : le sang complet et les tissus (homogénéisats) doivent être déprotéinisés et filtrés/centrifugés avant chargement sur les colonnes de SPE. L'urine, quant à elle, ne requiert habituellement qu'une étape de dilution et/ou de centrifugation. De nouvelles procédures d'extraction en phase solide pour l'analyse toxicologique systématique (STA) ont été décrites pour LC-MS (32). Dans cette dernière référence, de nombreuses phases d'adsorption SPE ont été évaluées afin de sélectionner la meilleure tant du point de vue du rendement d'extraction que de la pureté des extraits. Selon les auteurs, la phase d'adsorption Isolute $\mathrm{C} 8 \mathrm{~s}$ 'avère être la plus appropriée.

La micro-extraction en phase solide (SPME) est une alternative récente à la SPE et à la LLE. La SPME est une technique exempte de solvant qui convient particulièrement pour les analytes relativement volatiles. Elle est basée sur l'adsorption de l'analyte sur une phase stationnaire recouvrant une fine tige de silice fondue. Elle a déjà été utilisée comme technique de préparation pour la détermination des phénothiazines dans les liquides biologiques par LC-MS/MS (41).

\section{Procédures de screening en LC-MS}

L'HPLC couplée à un spectromètre de masse simple quadripôle ou en tandem est un appareil de plus en plus utilisé en routine, tout particulièrement pour l'analyse d'échantillons sanguins et plasmatiques $(1,10,13-18$, $21,24,25,28,30,42-44)$. Cependant, le développement de procédures de screening par LC-MS doit intégrer plusieurs limitations, comme indiqué par tous les experts dans le domaine $(1,13,15,43,45,46)$. Le contenu informatif des spectres obtenus en ionisation électrospray (ESI) et/ou en ionisation chimique à pression atmosphérique (APCI) est plus limité que celui des spectres obtenus par impact électronique (EI). La dissociation induite par collision (CID) obtenue en augmentant le voltage dit d'orifice ou du fragmenteur, permet d'obtenir des fragments structurellement intéressants. Les appareils modernes permettent d'alterner très rapidement l'acquisition de données spectrales obtenues sous des voltages différents au cours d'une même injection. De la sorte, des composés aux propriétés très différentes peuvent être analysés dans la même injection chromatographique sans perte d'information spectrale. La fragmentation peut cependant varier considérablement d'un appareil à l'autre (45-48).
Weinmann et coll. (45) ont montré que différents types d'appareils pouvaient générer des spectres de masse électrospray obtenus par dissociation induite par collision (CID) relativement reproductibles et comparables, si chaque appareil avait été préalablement testé et réglé à l'aide de composés-tests tels que l'halopéridol, le paracétamol, le métronidazole ou le métamizole. Ils en ont conclu que l'utilisation d'une librairie spectrale (électrospray-CD-MS) obtenue sur un ou deux instruments était transposable à un autre instrument après ajustement des paramètres énergétiques de la dissociation induite par collision (CID) à l'aide d'au minimum deux composés-tests. D'autre part, Gergov et coll. (49) ont étudié la reproductibilité des spectres de masse d'ions-fils obtenus à l'aide de différents appareils de LC-MS/MS en concluant, qu'après normalisation des conditions expérimentales, les bibliothèques spectrales LC-MS/MS de médicaments et de drogues étaient échangeables entre différents laboratoires. Les critères d'identification de composés par HPLC-MS à simple quadripôle ou en tandem ont été récemment passés en revue par Rivier (46). L'auteur de cette revue partage la conclusion finale de Rivier : la responsabilité finale incombe au toxicologue analyste de décider, selon les cas, des critères minimums nécessaires à la confirmation de l'identité d'un composé.

Un autre problème important de l'ionisation électrospray (ESI) est la suppression d'ions, à savoir la réduction de l'ionisation d'un analyte par les composés coéluants $(33-38,50,51)$. Dans ce type de situation, un toxique important pourrait s'avérer indétectable. A l'extrême, ce problème pourrait mener indirectement au décès du patient. La suppression d'ions résulte de la présence de composés moins volatils qui peuvent affecter l'efficacité de formation ou l'évaporation des gouttelettes de phase mobile. Au final, ce phénomène affecte la quantité d'ions chargés en phase gazeuse à même d'atteindre le détecteur. Les sels, les composés formant des paires d'ions, les substances endogènes, les médicaments et drogues ainsi que leurs métabolites de même que les standards internes deutérés sont responsables de suppression d'ionisation $(33,34)$. La suppression d'ionisation peut affecter négativement la limite de détection (LOD), augmenter le risque de résultats faussement négatifs, diminuer la précision et augmenter ainsi l'incertitude de mesure.

Dams et coll. (35) ont évalué la synergie entre type d'ionisation, technique de préparation de l'échantillon et type de liquide biologique (urine, salive et plasma) sur la survenue des effets de matrice. Ils ont constaté que les deux techniques d'ionisation, ESI et APCI, présentent des effets de matrice, l'ESI y étant malgré tout beaucoup plus sensible. La préparation de l'échantillon 
pourrait elle-même sinon réduire (purification), sinon amplifier (préconcentration) les effets de matrice.

Liang et coll. (34) ont montré que les neuf composés testés et leurs standards internes deutérés respectifs se suppriment l'un l'autre en ionisation électrospray (ESD). Au contraire, en mode d'ionisation APCI, sept des neuf composés ont vu leur ionisation augmentée par leurs propres standards internes. La suppression ou l'augmentation mutuelle de l'ionisation peut influencer les paramètres de sensibilité, de reproductibilité, d'exactitude et de linéarité en analyse quantitative par LC-MS ou LMC-MS/MS. Selon l'auteur, les procédures bio-analytiques utilisant l'ESI LC-MS ne devraient être employées en routine et acceptées pour publication dans les journaux scientifiques qu'à la condition que des études de suppression d'ions aient été réalisées. De telles investigations doivent inclure au minimum la comparaison des signaux obtenus d'une part avec l'analyte pur et, d'autre part, l'analyte ajouté à l'échantillon biologique préparé. Une approche plus complète passe par l'injection post-colonne de l'analyte afin d'évaluer les effets prolongés de la suppression d'ionisation (33). Si une suppression d'ions importante est détectée, des modifications doivent être apportées à la procédure (purification de l'échantillon, conditions chromatographiques, changement de réactifs, standardisation interne).

Plusieurs concepts ont été développés pour le screening et la détection en LC-MS. Afin de simplifier le choix d'une méthode adaptée et à même d'apporter des solutions à un problème analytique réel, les caractéristiques de base des procédures pour le screening urinaire sont reprises dans le tableau I, pour le screening sanguin/sérique dans le tableau II et pour le screening plasmatique avec quantification validée dans le tableau III. Bogusz (52), Rittner et coll. (44), Marquet et coll. (24, $43)$, et Maurer et coll. $(16,21,25,26,53)$ ont développé des procédures de screening sanguin basées sur un appareillage LC-MS à simple quadripôle, tandis que Gergov et coll. (18), Marquet et coll. (22) ainsi que Decaestecker et coll. (29) ont développé des procédures utilisant la LC-MS/MS. Gergov et coll. (18) ont décrit un screening plasmatique multi-composé en utilisant la LC-MS/MS triple quadripôle classique, tandis que Marquet et coll. (22), dans une étude préliminaire, ont comparé une procédure plus sensible utilisant un couplage quadripôle linéaire à trappe d'ions (Q-IT), à leur propre procédure en simple quadripôle mentionnée plus haut. Decaestecker et coll. (29) ont développé une procédure de screening urinaire en utilisant un couplage spectromètres de masse quadripôlaire et à temps de vol (Q-TOF). Gergov et coll. (30) ainsi que Pelander et coll. (31) ont développé des procédures de screening urinaire à l'aide d'un spectromètre de masse à temps de vol (TOF), procédure basée sur la mesure de la masse exacte et des temps de rétention et leur comparaison avec des données de référence, comme ce qui avait été réalisé sur GC-MS vingt cinq ans auparavant. Les auteurs ont conclu que la procédure était bien adaptée à l'identification provisoire sans substances de référence. Les quelques faux positifs soulignent l'importance et la nécessité de chacun des trois paramètres que sont

Tableau I : Procédures de LC-MS pour le screening ciblé de médicaments/drogues et/ou de leurs métabolites dans l'urine (U), le sang (S) et les cheveux (Ch).

\begin{tabular}{|l|l|l|l|l|}
\hline Composés & Échantillon Préparation & Mode de détection & Réf. \\
\hline B - Bloquants & U & SPE & ESI +, MS-MS, MRM & $(54)$ \\
\hline B- Bloquants & U & Hydrolyse enzymatique, LLE & APCI +, MS-MS, suivi de réactions multiples (MRM) & $(39)$ \\
\hline B 2-Agonistes & U & Hydrolyse enzymatique, LLE & ESI +, MS-MS, MRM & $(23)$ \\
\hline Neuroleptiques & U, S, Ch & $\begin{array}{l}\text { B et cheveux : SPE } \\
\text { U : Hydrolyse enzymatique, } \\
\text { pas d'extraction }\end{array}$ & ESI +, MS-MS, MRM & $(20)$ \\
\hline 637 médicaments & U & Hydrolyse enzymatique, SPE & TOF, HRMS & \\
\hline 433 médicaments & U & SPE & TOF, HRMS & $(31)$ \\
\hline Phénothiazines & U, S & SPME & ESI +, MS scan, MS-MS, MRM & $(30)$ \\
\hline Diurétiques & U & SPE & ESI -, MS-MS, MRM & $(41)$ \\
\hline Diurétiques & U & LLE & $(11)$ \\
\hline Drogues d'abus & U & LLE & QSI +l-, MS scan & $(12)$ \\
\hline Drogues d'abus & U & $\begin{array}{l}\text { Screening : injection directe } \\
\text { Confirmation : SPE }\end{array}$ & APCI +, MS-MS, MRM & $(29)$ \\
\hline
\end{tabular}


Tableau II : Procédures LC-MS pour le screening ciblé de médicaments/drogues et/ou de leurs métabolites en sang total (ST) ou sérum $(S)$.

\begin{tabular}{|l|l|l|l|l|}
\hline Composés & Echantillon & Préparation & Mode de détection & Réf. \\
\hline $\begin{array}{l}\text { Amphétamines, benzodiazépines } \\
\text { hallucinogènes, opiacés; cocaïne, } \\
\text { Olanzapine }\end{array}$ & $\begin{array}{l}\text { ST, S } \\
\text { (U) }\end{array}$ & SPE & APCI +, SIM pour le screening & $(52)$ \\
\hline $\begin{array}{l}\text { Amphétamines, benzodiazépines, } \\
\text { hallucinogènes, opiacés, hypnotiques, } \\
\text { Neuroleptiques }\end{array}$ & S & SPE & ESI +, MS scan & $(44)$ \\
\hline Médicaments sérotoninergiques & ST & LLE & APCI +, MS scan & \\
\hline Drogues d'abus & ST & SPE & Q-TOF, ESI +, MS et MS-MS alternées & $(29)$ \\
\hline Antihistaminiques & ST & LLE & ESI +, MS-MS, MRM & $(18)$ \\
\hline 238 médicaments & ST & LLE & ESI +, MS-MS, MRM & $(18)$ \\
\hline Antagonistes du calcium & S & SPE & ESI +, MS-MS, MRM & $(55)$ \\
\hline $\begin{array}{l}\text { Antidépresseurs, benzodiazépines, } \\
\text { médicaments cardiaques, opiacés } \\
\text { hypnotiques, neuroleptiques }\end{array}$ & S & SPE & ESI +/-, MS scan & $(24 ; 43)$ \\
\hline Myorelaxants - curarisants & ST & Déprotéinisation & ESI +, SIM pour la quantification & $(56)$ \\
\hline
\end{tabular}

Tableau III : Procédures LC-MS validées pour le screening ciblé et la quantification de médicaments/drogues dans le plasma (P).

\begin{tabular}{|l|l|l|l|l|l|}
\hline Compesés & Échantillon & Préparation & Mode de détection & Réf. \\
\hline $\begin{array}{l}\text { Anesthésiques, hypnotiques actifs } \\
\text { faible conc, et opiacés }\end{array}$ & P & LLE & $\begin{array}{l}\text { APCI +, MS Scan pour le screening, } \\
\text { MS en mode SIM pour la quantification }\end{array}$ & $(53)$ \\
\hline Benzodiazépines & P & LLE & $\begin{array}{l}\text { APCI +, MS Scan pour le screening, } \\
\text { MS en mode SIM pour la quantification }\end{array}$ & $(25)$ \\
\hline Antidiabétiques sulfonylurées & P & LLE & $\begin{array}{l}\text { APCI +, MS Scan pour le screening, } \\
\text { MS en mode SIM pour la quantification }\end{array}$ & $(16)$ \\
\hline B- Bloquants & P & SPE & $\begin{array}{l}\text { APCI +, MS Scan pour le screening, } \\
\text { MS en mode SIM pour la quantification }\end{array}$ & $(26)$ \\
\hline Neuroleptiques & P & SPE & $\begin{array}{l}\text { APCI +, MS Scan pour le screening, } \\
\text { MS en mode SIM pour la quantification }\end{array}$ & $(21)$ \\
\hline
\end{tabular}

la masse exacte, le temps de rétention et le schéma métabolique, pour permettre une identification définitive. Parallèlement à ces procédures générales, certaines procédures en LC-MS ou LC-MS/MS sont plus spécifiques pour le screening d'une classe de médicaments et de drogues d'abus $(29,40)$, médicaments sérotoninergiques (19), phénothiazines (41), neuroleptiques (20), $\beta_{2}$-agonistes (23), $\beta$-bloquants $(39,54)$, antagonistes du calcium $(55)$, diurétiques $(11,12)$, et myorelaxants-curarisants (56).

Maurer et coll. ont développé des procédures universelles en LC-MS simple quadripôle pour le screening, l'identification via librairies spectrales et, contrairement aux autres procédures de screening, la quantifica- tion plasmatique entièrement validée de nombreuses classes de médicaments $(16,21,25,26,53)$. Cette procédure générale en LC-MS est basée sur l'extraction liquide-liquide (LLE) standard $(16,25,28,53)$ ou SPE $(21,26)$ des auteurs. Ces procédures sont également utilisées pour les analyses en GC-MS (57-59). La séparation standard est basée sur un gradient rapide conduisant à des analyses d'une durée de 5 à 10 minutes. Le mode d'ionisation APCI a été préféré au mode électrospray pour les raisons évoquées ci-dessus. D'ailleurs, l'appareillage LC-MS utilisé avait une sensibilité plus importante en mode d'APCI. Les spectres de masse ont été enregistrés à deux voltages de fragmenteur ( $100 \mathrm{~V}$ et $200 \mathrm{~V})$ avec un cycle très court d'ac- 
quisition de données. Cependant, comme il en a déjà été discuté ci-dessus, nous devons garder à l'esprit que les mêmes voltages de fragmenteur utilisés dans des appareils différents peuvent induire des fragments d'abondances différentes (45-48). Par conséquent, chaque utilisateur doit choisir le(s) voltage(s) de fragmenteur le(s) plus adapté(s) à son propre appareil de manière à générer des spectres de masse les plus riches en information et les plus proches de ceux publiés (16, $21,25,26,53)$. Selon l'expérience de l'auteur sur trois différents appareils du même type simple quadripôle (LC-MS), cette démarche a permis l'utilisation correcte de la procédure de screening. En général, après screening et identification en mode scan à l'aide de la nouvelle librairie spectrale LC-MS des auteurs (60), les composés des classes de médicaments étudiées peuvent être quantifiés en mode d'ions sélectionnés (16, 21, 25, $26,53)$. Toutes les procédures de quantification ont été entièrement validées conformément aux recommandations internationales (61-63). Seules ces données de validation peuvent convaincre le lecteur de la fiabilité d'une procédure. Chaque méthode de dosage s'est avérée être spécifique et linéaire pour des concentrations allant du sub-thérapeutique au supra-thérapeutique et ce, pour la plupart des composés.

\section{Conclusions et perspectives}

La LC-MS se présente donc comme le complément idéal au standard analytique actuel qu'est la GC-MS, tout particulièrement pour la détection dans le plasma de médicaments plus polaires, instables ou faiblement dosés. Elle pourrait devenir un standard analytique en toxicologies clinique et médico-légale ainsi que dans l'analyse anti-dopage, pour autant que l'appareillage soit nettement moins coûteux, que les inconvénients actuels comme le manque de reproductibilité de la fragmentation, la réduction d'ionisation par la matrice, etc...soient surmontés, et pour terminer, qu'une seule des différentes techniques d'ionisation devienne le standard à utiliser.

\section{Remerciements}

L'auteur remercie les Docteurs Frank T. Peters et Denis $S$. Theobald pour leurs discussions très utiles.

Ce texte, initialement écrit en anglais, a été traduit avec l'accord de l'auteur, par Thierry Gougnard (Liège), Alain Verstraete (Gand) et Marc Deveaux (Paris), qui ont fait le maximum pour rester fidèles au style de l'auteur.

\section{Références}

1. Maurer $\mathrm{H}$. H. Position of chromatographic techniques in screening for detection of drugs or poisons in clinical and forensic toxicology and/or doping control [review]. Clin. Chem. Lab. Med. 2004 ; 42 : 1310-24.

2. Pelander A., Ojanpera I., Sistonen J., Rasanen I., Vuori E. Screening for basic drugs in 2-mL urine samples by dualplate overpressured layer chromatography and comparison with gas chromatography-mass spectrometry. J Anal. Toxicol. $2003 ; 27: 226-32$.

3. Lacassie E., Gaulier J.-M., Marquet P., Rabatel J.-F., Lachatre G. Methods for the determination of seven selective serotonin reuptake inhibitors and three active metabolites in human serum using high-performance liquid chromatography and gas chromatography. J Chromatogr. B Biomed. Sci. Appl. $2000 ; 742$ : 229-38.

4. Rasanen I., Ojanpera I., Vuori E. Quantitative screening for benzodiazepines in blood by dual-column gas chromatography and comparison of the results with urine immunoassay. J Anal. Toxicol. 2000 ; 24 : 46-53.

5. Rasanen I., Kontinen I., Nokua J., Ojanpera I., Vuori E. Precise gas chromatography with retention time locking in comprehensive toxicological screening for drugs in blood. J Chromatogr. B Analyt. Technol. Biomed. Life Sci. $2003 ; 788: 243-50$.

6. Kueh A. J., Marriott P. J., Wynne P. M., Vine J. H. Application of comprehensive two-dimensional gas chromatography to drugs analysis in doping control. J Chromatogr. A $2003 ; 1000: 109-24$.

7. Thormann W. Progress of electrokinetic techniques in therapeutic drug monitoring and in clinical and forensic toxicology [review]. Ther. Drug Monit. 2002 ; 24 : 222-31.

8. Makino K., Yano T., Maiguma T., Teshima D., Sendo T., Itoh Y., Oishi R. A rapid and simultaneous determination of several analgesic antiinflammatory agents by capillary zone electrophoresis. Ther. Drug Monit. 2003 ; 25 : 57480.

9. Van Bocxlaer J. F., Clauwaert K. M., Lambert W. E., Deforce D. L., Van den Eeckhout E. G., de-Leenheer A. P. Liquid chromatography-mass spectrometry in forensic toxicology [review]. Mass Spectrom. Rev. 2000; $19: 165-214$.

10. Gergov M., Robson J. N., Ojanpera I., Heinonen O. P., Vuori E. Simultaneous screening and quantitation of 18 antihistamine drugs in blood by liquid chromatography ionspray tandem mass spectrometry. Forensic Sci. Int. $2001 ; 121: 108-15$.

11. Thieme D., Grosse J., Lang R., Mueller R. K., Wahl A. Screening, confirmation and quantification of diuretics in urine for doping control analysis by high-performance liquid chromatography- atmospheric pressure ionisation tandem mass spectrometry. J. Chromatogr. B Biomed. Sci. Appl. $2001 ; 757: 49-57$.

12. Deventer K., Delbeke F. T., Roels K., Van Eenoo P. Screening for 18 diuretics and probenecid in doping analysis by liquid chromatography-tandem mass spectrometry. Biomed. Chromatogr. 2002 ; 16 : 529-35.

13. Marquet P. Progress of LC-MS in clinical and forensic toxicology [review]. Ther. Drug Monit. 2002 ; 24 : 255-76.

14. Marquet P. Is LC-MS suitable for a comprehensive scree- 
ning of drugs and poisons in clinical toxicology ? Ther. Drug Monit. 2002 ; 24 : 125-33.

15. Maurer H. H., Kraemer T., Kratzsch C., Peters F. T., Weber A. A. Negative ion chemical ionization gas chromatography-mass spectrometry (NICI-GC-MS) and atmospheric pressure chemical ionization liquid chromatography-mass spectrometry (APCI-LC-MS) of low-dosed and/or polar drugs in plasma. Ther. Drug Monit. 2002 ; 24 : 117-24.

16. Maurer H. H., Kratzsch C., Kraemer T., Peters F. T., Weber A. A. Screening, library-assisted identification and validated quantification of oral antidiabetics of the sulfonylureatype in plasma by atmospheric pressure chemical ionization liquid chromatography-mass spectrometry (APCILC-MS). J. Chromatogr. B Analyt. Technol. Biomed. Life Sci. $2002 ; 773: 63-73$.

17. Weinmann W., Muller C., Vogt S., Frei A. LC-MS-MS analysis of the neuroleptics clozapine, flupentixol, haloperidol, penfluridol, thioridazine, and zuclopenthixol in hair obtained from psychiatric patients. J. Anal. Toxicol 2002 ; $26: 303-7$.

18. Gergov M., Ojanpera I., Vuori E. Simultaneous screening for 238 drugs in blood by liquid chromatography-ion spray tandem mass spectrometry with multiple-reaction monitoring. J Chromatogr. B Analyt. Technol. Biomed. Life Sci. $2003 ; 795: 41-53$.

19. Goeringer K. E., McIntyre M., Drummer O. H. LC-MS analysis of serotonergic drugs. J. Anal. Toxicol. 2003 ; $27: 30-5$.

20. Josefsson M., Kronstrand R., Andersson J., Roman M. Evaluation of electrospray ionisation liquid chromatography-tandem mass spectrometry for rational determination of a number of neuroleptics and their major metabolites in human body fluids and tissues. J Chromatogr. B Analyt. Technol. Biomed. Life Sci. 2003 ; 789 : 151-67.

21. Kratzsch C., Weber A. A., Peters F. T., Kraemer T., Maurer H. H. Screening, library-assisted identification and validated quantification of fifteen neuroleptics and three of their metabolites in plasma by liquid chromatography/mass spectrometry with atmospheric pressure chemical ionization. J. Mass Spectrom. 2003 ; 38 : 283-95.

22. Marquet P., Saint-Marcoux F., Gamble T. N., Leblanc J. C. Comparison of a preliminary procedure for the general unknown screening of drugs and toxic compounds using a quadrupole-linear ion-trap mass spectrometer with a liquid chromatography-mass spectrometry reference technique. J. Chromatogr. B Analyt. Technol. Biomed. Life Sci. $2003 ; 789: 9-18$.

23. Thevis M., Opfermann G., Schanzer W. Liquid chromatography/electrospray ionization tandem mass spectrometric screening and confirmation methods for beta2-agonists in human or equine urine. J. Mass Spectrom. 2003 ; 38 : 1197-206.

24. Venisse N., Marquet P., Duchoslav E., Dupuy J. L., Lachatre G. A general unknown screening procedure for drugs and toxic compounds in serum using liquid chromatography-electrospray-single quadrupole mass spectrometry. J. Anal. Toxicol. $2003 ; 27: 7-14$.

25. Kratzsch C., Tenberken O., Peters F. T., Weber A. A., Kraemer T., Maurer H. H. Screening, library-assisted identification and validated quantification of twentythree benzodiazepines, flumazenil, zaleplone, zolpidem and zopiclone in plasma by liquid chromatography/mass spec- trometry with atmospheric pressure chemical ionization. J. Mass Spectrom. 2004 ; 39 : 856-72.

26. Maurer H. H., Tenberken O., Kratzsch C., Weber A. A., Peters F. T. Screening for, library-assisted identification and fully validated quantification of twenty-two beta-blockers in blood plasma by liquid chromatography-mass spectrometry with atmospheric pressure chemical ionization. J. Chromatogr. A 2004 ; 1058 : 169-81.

27. Maurer H. H. Screening procedures for simultaneous detection of several drug classes used in the high throughput toxicological analysis and doping control [review]. Comb. Chem. High Throughput Screen. 2000 ; 3 : 461-74.

28. Maurer H. H., Kratzsch C., Weber A. A., Peters F. T., Kraemer T. Validated assay for quantification of oxcarbazepine and its active dihydro metabolite 10 -hydroxy carbazepine in plasma by atmospheric pressure chemical ionization liquid chromatography/mass spectrometry. J. Mass Spectrom. 2002 ; 37 : 687-92.

29. Decaestecker T. N., Clauwaert K. M., Van Bocxlaer J. F., Lambert W. E., Van den Eeckhout E. G., Van Peteghem C. H., De Leenheer A. P. Evaluation of automated single mass spectrometry to tandem mass spectrometry function switching for comprehensive drug profiling analysis using a quadrupole time-of-flight mass spectrometer. Rapid Commun. Mass Spectrom. 2000 ; 14 : 1787-92.

30. Gergov M., Boucher B., Ojanpera I., Vuori E. Toxicological screening of urine for drugs by liquid chromatography/time-of-flight mass spectrometry with automated target library search based on elemental formulas. Rapid Commun. Mass Spectrom. 2001 ; 15 : 521-6.

31. Pelander A., Ojanpera I., I, Laks S., Rasanen I., Vuori E. Toxicological Screening with Formula-Based Metabolite Identification by Liquid Chromatography/Time-of-Flight Mass Spectrometry. Anal. Chem. 2003 ; 75 : 5710-8.

32. Decaestecker T. N., Coopman E. M., Van Peteghem C. H., Van Bocxlaer J. F. Suitability testing of commercial solidphase extraction sorbents for sample clean-up in systematic toxicological analysis using liquid chromatography(tandem) mass spectrometry. J. Chromatogr. B Analyt. Technol. Biomed. Life Sci. 2003 ; 789 : 19-25.

33. Annesley T. M. Ion suppression in mass spectrometry. Clin. Chem. 2003 ; 49 : 1041-4.

34. Liang H. R., Foltz R. L., Meng M., Bennett P. Ionization enhancement in atmospheric pressure chemical ionization and suppression in electrospray ionization between target drugs and stable-isotope-labeled internal standards in quantitative liquid chromatography/tandem mass spectrometry. Rapid Commun. Mass Spectrom. 2003 ; 17 : 2815-21.

35. Dams R., Huestis M. A., Lambert W. E., Murph, C. M. Matrix effect in bio-analysis of illicit drugs with LCMS/MS : influence of ionization type, sample preparation, and biofluid. J Am. Soc. Mass Spectrom. $2003 ; 14$ : 1290-4.

36. Muller C., Schafer P., Stortzel M., Vogt S., Weinmann W. Ion suppression effects in liquid chromatography-electrospray- ionisation transport-region collision induced dissociation mass spectrometry with different serum extraction methods for systematic toxicological analysis with mass spectra libraries. J. Chromatogr. B Analyt. Technol. Biomed. Life Sci. 2002 ; 773 : 47-52.

37. Mortier K. A., Clauwaert K. M., Lambert W. E., Van Bocxlaer J. F., Van den Eeckhout E. G., Van Peteghem C. H., De Leenheer A. P. Pitfalls associated with liquid chro- 
matography/electrospray tandem mass spectrometry in quantitative bioanalysis of drugs of abuse in saliva. Rapid Commun. Mass Spectrom. 2001 ; 15 : 1773-5.

38. King R., Bonfiglio R., Fernandez-Metzler C., Miller-Stein C., Olah T. Mechanistic investigation of ionization suppression in electrospray ionization. J. Am. Soc. Mass Spectrom. $2000 ; 11: 942-50$.

39. Thevis M., Opfermann G., Schanzer W. High speed determination of beta-receptor blocking agents in human urine by liquid chromatography/tandem mass spectrometry. Biomed. Chromatogr. 2001 ; 15 : 393-402.

40. Nordgren H. K., Beck O. Multicomponent screening for drugs of abuse : direct analysis of urine by LC-MS-MS. Ther. Drug Monit. 2004 ; 26 : 90-7.

41. Kumazawa T., Seno H., Watanabe-Suzuki K., Hattori H., Ishii A., Sato K., Suzuki O. Determination of phenothiazines in human body fluids by solid-phase microextraction and liquid chromatography/tandem mass spectrometry. J. Mass Spectrom. 2000; 35: 1091-9.

42. Maurer H. H., Schmitt C. J., Weber A. A., Kraemer T. Validated electrospray LC-MS assay for determination of the mushroom toxins alpha- and beta-amanitin in urine after immunoaffinity extraction. J. Chromatogr. B Biomed. Sci. Appl. 2000 ; 748 : 125-35.

43. Saint-Marcoux F., Lachatre G., Marquet P. Evaluation of an improved general unknown screening procedure using liquid chromatography-electrospray-mass spectrometry by comparison with gas chromatography and high-performance liquid-chromatography-diode array detection. J. Am. Soc. Mass Spectrom. 2003 ; 14 : 14-22.

44. Rittner M., Pragst F., Bork W. R., Neumann J. Screening method for seventy psychoactive drugs or drug metabolites in serum based on high-performance liquid chromatography-electrospray ionization mass spectrometry. J. Anal. Toxicol $2001 ; 25: 115-24$.

45. Weinmann W., Stoertzel, M., Vogt, S., Wendt, J. Tune compounds for electrospray ionisation/in-source collisioninduced dissociation with mass spectral library searching. J. Chromatogr. A 2001 ; 926 : 199-209.

46. Rivier L. Criteria for the identification of compounds by liquid chromatography-mass spectrometry and liquid chromatography-multiple mass spectrometry in forensic toxicology and doping analysis. Anal. Chim. Acta 2003; 492 : 69-82.

47.Lips A.G.A.M., Lameijer W., Fokkens R. H., Nibbering N.M.M. Methodology for the development of a drug library based upon collision-induced fragmentation for the identification of toxicologically relevant drugs in plasma samples. J. Chromatogr. B Biomed. Sci. Appl. 2001 ; $759: 191-207$.

48. Hough J. M., Haney C. A., Voyksner R. D., Bereman R. D. Evaluation of electrospray transport CID for the generation of searchable libraries. Anal. Chem. $2000 ; 72$ : 2265-70.

49. Gergov M., Weinmann W., Meriluoto J., Uusitalo J., Ojanpera I. Comparison of product ion spectra obtained by liquid chromatography/triple-quadrupole mass spectrometry for library search. Rapid Commun. Mass Spectrom. $2004 ; 18: 1039-46$.

50. Mallet C. R.,'Lu Z., Mazzeo J. R. A study of ion suppression effects in electrospray ionization from mobile phase additives and solid-phase extracts. Rapid Commun. Mass Spectrom. 2004 ; 18 : 49-58.
51. Sojo L. E., Lum G., Chee P. Internal standard signal suppression by co-eluting analyte in isotope dilution LC-ESIMS. Analyst $2003 ; 128: 51-4$.

52. Bogusz M. J. Liquid chromatography-mass spectrometry as a routine method in forensic sciences: a proof of maturity. J. Chromatogr. B Biomed. Sci. Appl. 2000 ; 748 : 3-19.

53. Kratzsch, C., Peters F. T., Kraemer T., Weber A. A., Maurer H. H. Simple APCI-LC-MS method for screening, libraryassisted identification and validated quantification of anesthetics, benzodiazepines and low dosed opioids in plasma often asked for in the context of the diagnosis of brain death. Pragst, F. and Aderjan, R. Proceedings of the XIIIth GTFCh Symposium in Mosbach, 299-309. 2003. Heppenheim (Germany), Helm-Verlag.

54. Gergov M., Robson J. N., Duchoslav E., Ojanpera I. Automated liquid chromatographic/tandem mass spectrometric method for screening beta-blocking drugs in urine. J Mass Spectrom. $2000 ; 35: 912-8$.

55. Mueller C. A., Gonzalez A. B., Weinmann W. Screening for dihydropyridine calcium channel blockers in plasma by automated solid-phase extraction and liquid chromatography/tandem mass spectrometry. J. Mass Spectrom. $2004 ; 39: 639-46$.

56. Sayer H., Quintela O., Marquet P., Dupuy J. L., Gaulier J. M., Lachatre G. Identification and quantitation of six nondepolarizing neuromuscular blocking agents by LC-MS in biological fluids. J Anal. Toxicol. 2004 ; 28 : 105-10.

57. Maurer, H. H. In Mass spectral and GC data of drugs, poisons, pesticides, pollutants and their metabolites, part 4 (Pfleger, K., Maurer, H. H., and Weber, A., Eds.) pp 3-241, Wiley-VCH, Weinheim, 2000.

58. Peters F. T., Kraemer T., Maurer H. H. Drug testing in blood : validated negative-ion chemical ionization gas chromatographic-mass spectrometric assay for determination of amphetamine and methamphetamine enantiomers and its application to toxicology cases. Clin. Chem. $2002 ; 48: 1472-85$

59. Peters F. T., Schaefer S., Staack R. F., Kraemer T., Maurer $H$. H. Screening for and validated quantification of amphetamines and of amphetamine- and piperazine-derived designer drugs in human blood plasma by gas chromatography/mass spectrometry. J. Mass Spectrom. 2003 ; 38 : 659-76.

60. Maurer, H. H., Weber A. A. APCI and Electrospray LCMS Library of Drugs, Poisons, Pesticides, Pollutants and their Metabolites Agilent Technologies, Palo Alto (CA), 2005.

61. Shah V. P., Midha K. K., Findlay J. W., Hill H. M., Hulse J. D., McGilveray I. J., McKay G., Miller K. J., Patnaik R. N., Powell M. L., Tonelli A., Viswanathan C. T., Yacobi A. Bioanalytical method validation - a revisit with a decade of progress. Pharm. Res. $2000 ; 17: 1551-7$.

62. Peters F. T., Maurer H. H. Bioanalytical method validation and its implications for forensic and clinical toxicology A review [review]. Accred. Qual. Assur. 2002 ; 7 : 441-9.

63. Peters, F. T. Applications of Liquid Chromatography-Mass Spectrometry in Toxicology (Polettini, A., Ed.), Pharmaceutical Press, London, in press, 2005. 